\title{
Anatomia e uso da madeira de duas variedades de Sclerolobium paniculatum Vog. do sul do Maranhão, Brasil ${ }^{1}$
}

\author{
Iran Paz Pires ${ }^{2}$ e Carmen Regina Marcati ${ }^{2,3}$
}

Recebido em 13/04/2004. Aceito em 01/03/2005

\begin{abstract}
RESUMO - (Anatomia e uso da madeira de duas variedades de Sclerolobium paniculatum Vog. do sul do Maranhão, Brasil). O presente estudo traz informações sobre a utilização da madeira de duas variedades de Sclerolobium paniculatum (var. subvelutinum e rubiginosum) em propriedades rurais do sul do Maranhão, a análise anatômica do lenho e a correlação destas características com o uso da madeira. Foram realizadas entrevistas com 17 agricultores residentes na zona rural dessa região, com a finalidade de se conhecer a utilização destas variedades nas propriedades rurais. As variedades subvelutinum Benth. e rubiginosum (Mart. ex Tul. ) Benth. são conhecidas na região como cachamorra-preta e cachamorra-branca, respectivamente, sendo a primeira mais utilizada na confecção de cercados. Para o estudo anatômico do lenho, foram coletados discos à altura do peito de três indivíduos de cada variedade e, destes, obtidas amostras do cerne, na região de transição com o alburno. As amostras foram processadas de acordo com a metodologia usual para anatomia de madeira. Houve diferença estatística significativa $(\mathrm{p}<0,05)$ para seis parâmetros anatômicos entre as duas variedades. A utilização diferencial das variedades pode ser decorrente, principalmente, das características das fibras e dos elementos de vasos. Sugere-se que sejam realizados estudos que abordem variações climáticas e características do solo para melhor entendimento das diferenças anatômicas quantitativas encontradas no lenho das variedades.
\end{abstract}

Palavras-chave:anatomia da madeira, Sclerolobium paniculatum var. rubiginosum, Sclerolobium paniculatum var. subvelutinum, uso da madeira

\begin{abstract}
Wood anatomy and use of two varieties of Sclerolobium paniculatum Vog. in the south of Maranhão State, Brazil). This work presents information about the use of wood of Sclerolobium paniculatum (var. subvelutinum and rubiginosum) at rural properties in the south of the state of Maranhão, Brazil, about the woods' anatomy and about the correlation between certain anatomical characteristics and the wood's practical use. Interviews were made with 17 farmers from this region in order to know how they use the two varieties in their properties. Regionally, the subvelutinum Benth. and rubiginosum (Mart. ex Tul. ) Benth. varieties are known as "cachamorra-preta" and "cachamorra-branca", respectively and the former is the most frequently applied, especially as fences. For the wood anatomy analyses, discs at the breast height were collected from three specimens of each variety. Next, samples were obtained from the discs core, and usual wood anatomy methodology was applied. The two varieties were statistically different $(\mathrm{p}<0,05)$ for six anatomical parameters. Fiber and vessel elements characteristics seem to be the most important features to explain the different use of the two varieties. It is suggested that more studies should be performed on climate variations and soil characteristics to get a better understanding about the causes of the wood anatomy quantitative differences found in this study.
\end{abstract}

Key words: wood anatomy, Sclerolobium paniculatum var. rubiginosum, Sclerolobium paniculatum var. subvelutinum, wood use

\section{Introdução}

Pertencente a família Leguminosae-Cesalpinioideae, Sclerolobium paniculatum var. rubiginosum (Mart. ex Tul.) Benth e $S$. paniculatum Vog. var. subvelutinum Benth. constituem um par vicariante e têm se mostrado particularmente abundantes na região dos cerrados, principalmente nos estados do Maranhão e do Piauí onde há áreas com grande densidade destas variedades (B.A.S. Pereira, dados não publicados; Almeida et al. 1998;
Carvalho 1994). As regiões dos cerrados são caracterizadas por apresentarem marcada pluviosidade sazonal, com invernos frios e secos (Franco 2002).

Sclerolobium paniculatum é espécie pioneira, colonizadora de terrenos marginais e margens de estradas, que freqüentemente inicia a sucessão secundária em áreas abertas pela germinação intensa de suas sementes no solo. Tem se destacado pelo rápido crescimento e sua madeira é amplamente utilizada por comunidades rurais do Centro-Oeste e Nordeste, na

\footnotetext{
1 Trabalho de Iniciação Científica do primeiro Autor

2 Faculdade de Ciências Agronômicas, Departamento de Recursos Naturais - Ciências Florestais, Universidade Estadual Paulista, Campus de Botucatu, C. Postal 237, CEP 18603-970, Botucatu, SP, Brasil

3 Autor para correspondência: carmen@fca.unesp.br
} 
confecção de mourões, esteios, embalagens e caibros, na construção civil, além de fonte para a produção de lenha e carvão vegetal, sendo inclusive indicada para plantios energéticos (Diniz 1982; Dias et al. 1992; Carvalho 1994; Lorenzi 1998; Franke 1999).

Observações preliminares apontam para a ampla utilização da madeira de $S$. paniculatum var. subvelutinum e $S$. paniculatum var. rubiginosum por comunidades rurais do sul do Maranhão (Pires, comunicação pessoal). Segundo o mesmo autor, os agricultores as diferenciam através da coloração da casca, branca a acinzentada, sendo mais escura na variedade subvelutinum. Apesar da grande utilização dessa madeira em propriedades rurais, seus estudos anatômicos têm sido limitados somente ao gênero sem indicação das espécies estudadas, com ocorrência no Pará (SUDAM 1981; Mainieri et al. 1983), e às variedades subvelutinum e rubiginosum com ocorrência no Distrito Federal (B.A.S. Pereira, dados não publicados).

Considerando a importância da madeira de S. paniculatum variedades subvelutinum e rubiginosum para as comunidades rurais do sul do Maranhão, buscou-se conhecer o seu uso nessas comunidades e estudar as características anatômicas e a densidade dessas madeiras na tentativa de correlacionar essas características com o seu uso.

\section{Material e métodos}

As plantas estudadas foram coletadas em área de cerrado localizado na Fazenda Ipanema situada cerca de $60 \mathrm{~km}$ da sede do município de Riachão, Estado do Maranhão. O município de Riachão tem como coordenadas geográficas $7^{\circ} 22^{\prime} 30^{\prime \prime} \mathrm{S}$ e $46^{\circ} 37^{\prime} 30^{\prime} \mathrm{W}$. Ocupa área de $6.373,153 \mathrm{~km}^{2}$, limita-se a oeste com o município de Carolina, a leste com o município de Balsas, a norte com o município de Feira Nova do Maranhão e Nova Colinas, e a sul com o município de Campos Lindo, este no Estado de Tocantins.

O clima da região caracteriza-se como tropical quente de seca atenuada ( $4 \mathrm{cth}$ ), em que a média do mês mais quente é $28,3^{\circ} \mathrm{C}$ e a média mensal mais fria é $26,1^{\circ} \mathrm{C}$, sendo a média anual de $26,5^{\circ} \mathrm{C}$ (Diniz 1982).

A região apresenta três meses mais chuvosos (janeiro a março) e cinco meses secos, não apresentando altitude superior à $200 \mathrm{~m}$, com formação geológica Pedra do Fogo (permiano inferior). Apresenta solos do tipo Latossolo Vermelho Amarelo e areias quartzosas vermelhas e amarelas (Diniz 1982).
Com a finalidade de se conhecer a utilização das variedades nas propriedades rurais, foram realizadas entrevistas com os agricultores da zona rural de RiachãoMA. Foram entrevistados todos os agricultores que residem em diferentes fazendas num raio de $20 \mathrm{~km}$, onde ocorre a espécie em estudo. Ao todo, foram entrevistadas 17 pessoas, as quais responderam questões relacionadas ao conhecimento e ao uso das variedades em sua propriedade (Tab. 1).

Para os estudos de densidade básica e anatomia do lenho, foram retirados discos a 1,30 $\mathrm{m}$ do solo, de três espécimes de $S$. paniculatum var. subvelutinum (Fig. 1) e de S. paniculatum var. rubiginosum (Fig. 2), que estavam sendo abatidas. Os discos, com $6 \mathrm{~cm}$ de espessura, foram retirados de indivíduos adultos,

Tabela 1. Modelo de formulário utilizado nas entrevistas com os proprietários rurais do sul do Maranhão, Brasil.

Nome:

Local:

Data:

Grau de escolaridade:

1 - Você conhece as árvores popularmente referidas como paupombo, cachamorra branca e cachamorra preta?

2 - Quais as principais utilizações que você atribui à cachamorra branca e cachamorra preta (lenha, construção civil, estaca, mourão,etc.)?

3 - Qual delas você mais utiliza em sua propriedade? Por que?

4 - Qual delas apresenta maior peso?

5 - Qual delas apresenta maior resistência às pragas (cupins, brocas, etc.)?

6 - Qual delas você utiliza quando tem necessidade de peças longas?

7 - Por quantos anos, mais ou menos, um mourão de cerca, destas espécies, dura em sua propriedade?

8 - Com que frequiência você observa uma ou outra variedade no campo?

9 - Em qual tipo de vegetação você já notou a presença de cachamorra branca e cachamorra preta? Dê algumas características desta vegetação.

10 - Qual delas, em sua opinião, é mais resistente ao corte de machado?

11 - Há uma idade e/ou tamanho preferencial para uma maior ou menor utilização da madeira de cachamorra branca e cachamorra preta?

12 - Há alguma característica incomum nessas árvores em relação às demais espécies que você conhece?

13 - Você costuma ver árvores de cachamorra rachadas, quebradas ou tombadas no campo? Qual delas?

14 - Além da cachamorra qual outra espécie de madeira que você mais utiliza em sua propriedade?

15 - Em sua opinião a cachamorra é uma madeira de crescimento rápido ou lento?

16 - Para o uso em sua propriedade, qual delas é a mais importante? 
distantes $20-50 \mathrm{~m}$ entre si. Foram obtidos a altura e o diâmetro à altura do peito (DAP) de dez árvores de cada variedade (Tab. 2) com auxílio de hipsômetro e suta, respectivamente, e as médias dos dados das duas variedades foram comparadas pelo teste de $\mathrm{F}$ ao nível de probabilidade de $5 \%$.

Ramos e folhas das árvores amostradas foram coletados. O material foi identificado no Laboratório de Anatomia Vegetal da Universidade de Brasília (UnB) pelo professor Dr. José Elias de Paula e colaboradores, a partir das exsicatas confeccionadas. As exsicatas e parte dos discos representativos das duas variedades encontram-se depositados no Herbário e na Xiloteca $\left(\mathrm{BOT}_{\mathrm{w}} 1315,1316,1317\right.$ para $S$. paniculatum var. rubiginosum e $\mathrm{BOT}_{\mathrm{w}} 1318,1319,1320$ para $S$. paniculatum var. subvelutinum) do Departamento de Recursos Naturais - Ciências Florestais da Faculdade de Ciências Agronômicas, Universidade Estadual Paulista (UNESP), Campus de Botucatu, Estado de São Paulo.

A determinação da densidade básica seguiu as recomendações da Associação Brasileira de Normas Técnicas (1940), com a averiguação do volume da madeira saturada pelo método hidrostático, utilizando-se água em lugar do mercúrio. $\mathrm{O}$ número pequeno de amostras da madeira de cada variedade para a análise de densidade básica $(n=3)$ impossibilitou a realização de análise estatística para essa variável.

Dos discos, foram obtidas amostras do cerne de $1,5 \mathrm{~cm}^{3}$, na região de transição para o alburno. Para as análises microscópicas, as amostras foram amolecidas por meio de cozimento e seccionadas em micrótomo de deslize para a obtenção dos cortes histológicos, que foram corados com safranina $1 \%$ em etanol $50 \%$ e coloração com azul de astra $0,5 \%$, juntamente com os corantes fucsina básica 5\% e acridinorange 1\% (Gerlach 1984; Roeser 1972; Kropp 1972). A fixação dos corantes foi feita com acetato de butila e as lâminas histológicas foram montadas permanentemente em Bálsamo do Canadá. A dissociação das células seguiu a metodologia de Franklin (1945).

Três espécimes de cada variedade foram analisados quanto à anatomia do lenho. Para as análises qualitativa e quantitativa foram seguidas as orientações propostas pelo IAWA Committee (1989). As análises quantitativas foram realizadas com as mensurações das seguintes variáveis: fibras (comprimento, diâmetro, diâmetro do lume), vasos (comprimento, diâmetro, freqüência, diâmetro das pontoações intervasculares), raios (frequiência/mm, altura em $\mu \mathrm{m}$, largura em $\mu \mathrm{m}$ ). As análises foram realizadas com o auxílio de microscópio de luz e ocular micrométrica. Os valores foram convertidos em micrômetros, empregando-se um fator de conversão. Os valores para os diversos parâmetros foram assim relacionados: (mínimo-) média (-máximo).

Foram realizadas 30 medições para cada característica anatômica e as médias dos dados das duas variedades foram comparadas pelo teste $\mathrm{F}$, ao nível de probabilidade de $5 \%$.

\section{Resultados}

Entrevista com os agricultores da região - Entre os agricultores há diversidade de denominações para o tipo de vegetação de ocorrência das variedades, a saber: capão, carrasco, cerrados, chapada argilosa, chapada arenosa, mas há uma predominância de chapada para $S$. paniculatum var. subvelutinum e capão para a $S$. paniculatum var. rubiginosum.

A partir dessas entrevistas, observou-se que todos conhecem S. paniculatum var. subvelutinum como cachamorra-preta e $S$. paniculatum var. rubiginosum como cachamorra-branca, em alusão às cores acinzentada e branca, respectivamente, características da sua casca.

Os agricultores utilizam, juntamente com a cachamorra-preta e a cachamorra-branca, outras espécies da região, sendo as mais citadas: capitão-docampo, candeia, aroeira, pequi, birro, angico, jatobá, sucupira-amarela, barbatimão, pau d'arco, araçá e bacuri. Quando comparado o crescimento das duas variedades de Sclerolobium no campo com as demais espécies, $100 \%$ dos entrevistados consideram que as variedades de cachamorra-preta e cachamorra-branca são de crescimento mais rápido.

Dentre os agricultores, $77 \%$ utilizam com mais freqüência a cachamorra-preta, entretanto as duas variedades são bastante consumidas nas propriedades rurais. A cachamorra-preta, identificada pelos agricultores como sendo a mais pesada e com maior resitência às pragas é utilizada para estacas, mourões e cercados (Fig. 3). Dos agricultores entrevistados, 70\% utilizam a madeira de cachamorra-branca como peças longas, pois esta variedade, segundo os mesmos, cresce mais em altura no campo quando comparada com a cachamorra-preta. $\mathrm{O}$ diâmetro e a altura das espécies estão identificados na Tab. 2.

Segundo os agricultores, a durabilidade natural da madeira de cachamorra-preta sem tratamento chega a 10 anos na propriedade, enquanto que a da cachamorrabranca chega a, no máximo, 6 anos. Além da durabilidade natural, todos eles avaliaram a cachamorra-preta como 
Tabela 2. Diâmetro (D) e altura (A), em metros, de dez árvores de Sclerolobium paniculatum var. subvelutinum Benth. e S. paniculatum var. rubiginosum (Mart. ex Tul.) Benth. da região de Riachão, MA, Brasil. Resultado da análise estatística com a probabilidade de significância entre as árvores $(\mathrm{p}<0,05)$.

\begin{tabular}{|c|c|c|c|c|c|c|c|c|c|c|c|c|c|c|c|c|c|c|c|c|c|}
\hline & \multicolumn{10}{|c|}{$\begin{array}{l}\text { S. paniculatum var. subvelutinum } \\
\text { (cachamorra-preta) }\end{array}$} & \multicolumn{11}{|c|}{$\begin{array}{l}\text { S. paniculatum var. rubiginosum } \\
\text { (cachamorra-branca) }\end{array}$} \\
\hline & 1 & 2 & 3 & 4 & 5 & 6 & 7 & 8 & 9 & 10 & 1 & 2 & 3 & 4 & 5 & 6 & 7 & 8 & 9 & 10 & Prob $>F$ \\
\hline $\mathrm{D}$ & 0,31 & 0,30 & 0,32 & 0,36 & 0,31 & 0,22 & 0,26 & 0,32 & 0,34 & 0,30 & 0,43 & 0,37 & 0,29 & 0,40 & 0,35 & 0,35 & 0,50 & 0,38 & 0,37 & 0,43 & 0,2858 \\
\hline A & 17 & 16 & 15 & 16,5 & 17 & 13 & 16,5 & 14 & 16,5 & 17 & 16 & 19,5 & 21 & 17 & 18,5 & 18,5 & 20 & 16,5 & 15,5 & 21 & 0,2805 \\
\hline
\end{tabular}
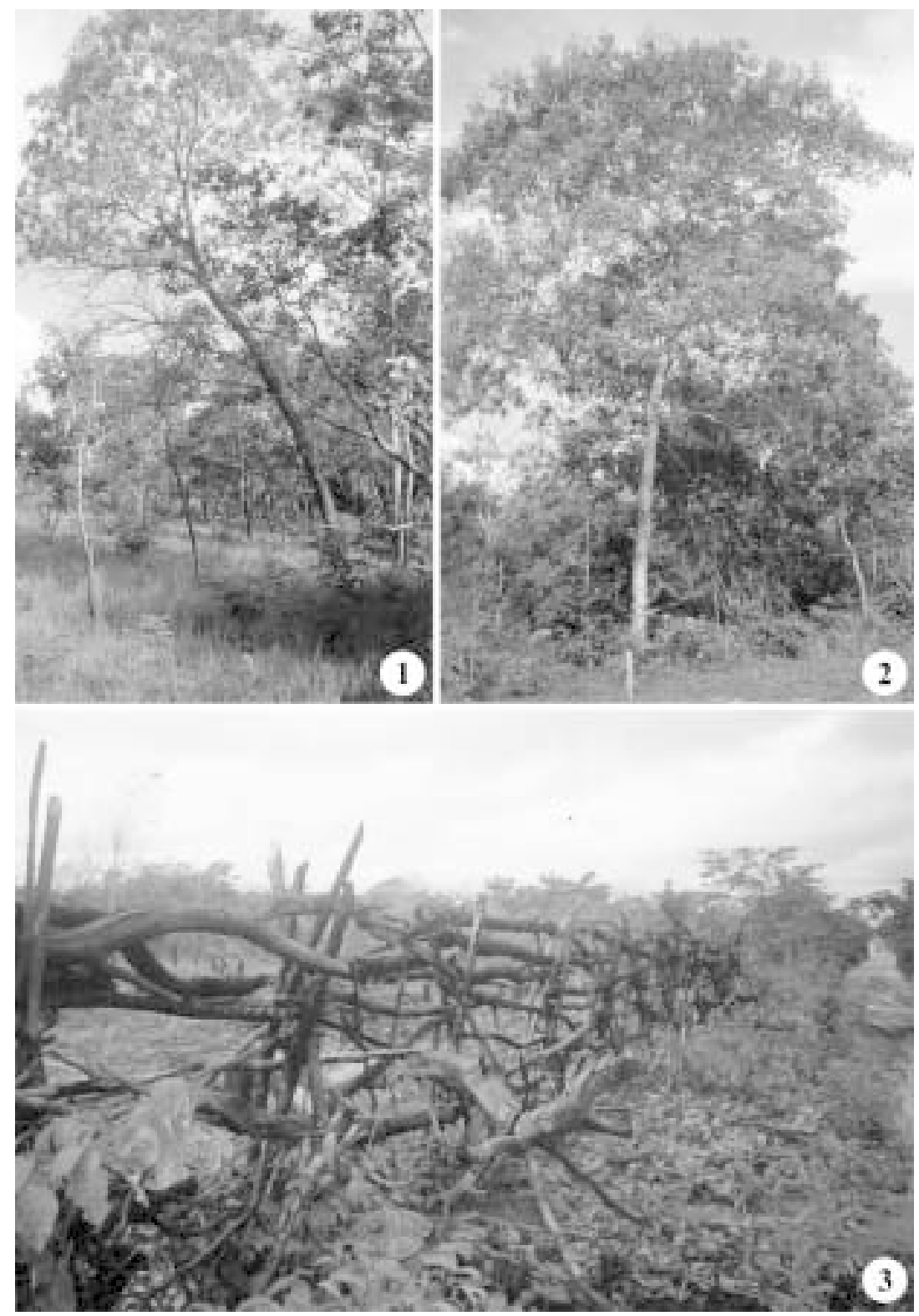

Figuras 1-3. Sclerolobium paniculatum Vog.1. Árvore de cachamorra-preta (S. paniculatum var. subvelutinum Benth.). 2. Árvore de cachamorra-branca (S. paniculatum var. rubiginosum (Mart. ex Tul.) Benth.). 3. Cerca de caiçara feita com galhada de cachamorrapreta (S. paniculatum var. subvelutinum). 
a mais resistente ao corte com machado e a mais importante para utilização geral nas propriedades.

No que diz respeito à freqüência de árvores rachadas, tombadas ou quebradas no campo, $71 \%$ dos agricultores entrevistados citaram como sendo mais freqüente a cachamorra-branca.

Densidade básica e anatomia da madeira das duas variedades - A Tab. 3 apresenta os dados quantitativos da densidade básica e das características anatômicas da madeira das duas variedades.

Qualitativamente, as espécies $S$. paniculatum var. subvelutinum e $S$. paniculatum var. rubiginosum não variam quanto à anatomia da madeira (Fig. 4-9).

O lenho das duas variedades caracteriza-se por apresentar: Camadas de crescimento - irregularmente demarcadas por zonas fibrosas (Fig. 4); Vasos - porosidade difusa (Fig. 4, 5), vasos solitários predominantes (43 e $36 \%$ em S. paniculatum var. subvelutinum e $S$. paniculatum var. rubiginosum, respectivamente), múltiplos de dois (20 e 19\%, respectivamente), de três ( 20 e $21 \%$, respectivamente), quatro (10 e $8 \%$, respectivamente), cinco ( 2 e $5 \%$, respectivamente) e seis (5 e $11 \%$, respectivamente), elementos de vasos sem apêndices (Fig. 10) e com apêndices, que se apresentam em uma (Fig. 11) ou em ambas as extremidades (Fig. 12, 13), placa de perfuração simples (Fig. 10-13), pontoações intervasculares alternas (Fig. 14) e guarnecidas (Fig. 15), pontoações radiovasculares com aréolas distintas e semelhantes às intervasculares em tamanho e forma; Fibras - com pontoações simples (Fig. 6, 7, 16, 17), Parênquima axial - paratraqueal vasicêntrico escasso (Fig. 4, 5) com duas a cinco células por série (Fig. 16, 17); Raios - exclusivamente unisseriados (99\%) (Fig. 6, $7,16,17)$ e homogêneos formados por células procumbentes (Fig. 8, 9).

Análise estatística das características anatômicas quantitativas - A análise estatística para os dez parâmetros entre as duas variedades de $S$. paniculatum está apresentada na Tab. 3. Entre as duas variedades, foram encontradas diferenças significativas $(p<0,05)$ para seis parâmetros a saber: freqüência e diâmetro de vasos; freqüência e largura dos raios; diâmetro e espessura da parede das fibras.

\section{Discussão}

A diversidade de denominações para o tipo de vegetação de ocorrência das variedades dadas pelos agricultores da região caracteriza fitofisionomias correspondentes ao cerrado, sendo que os tipos chapada para S. paniculatum var. subvelutinum e capão para $S$. paniculatum var. rubiginosum sugerem que as variedades ocorram em planície de vegetação rasa e em porção de vegetação isolada no meio do campo (manchas), respectivamente (Pires, comunicação pessoal). Estes dados estão de acordo com a literatura para a ocorrência das variedades (Ribeiro \& Walter 1998).

Neste trabalho pôde-se observar que os agricultores entrevistados conhecem as espécies pelo nome comum cachamorra, entretanto, na literatura, apenas Carvalho (1994) se refere ao nome cachamorra para o gênero Sclerolobium e este nome, segundo o autor, é usado apenas no Estado do Piauí. A espécie S. paniculatum também é conhecida por outros nomes comuns, dependendo da região em que ocorre: pau-pombo (PI, MA), arapacu, cangalheiro (MG), carvão-de-ferreiro (PA, BA, MG), carvoeiro (DF, GO, MG), carvoeirodo-cerrado (DF), passariúva (SP), táxi-branco-de-terra-

Tabela 3. Dados quantitativos de densidade básica e anatômicos das duas variedades de Sclerolobium paniculatum estudadas e resultado da análise estatística das características anatômicas entre as variedades com a probabilidade de significância de cada variável (p<0,05).

\begin{tabular}{|c|c|c|c|}
\hline Variáveis & var. subvelutinum & var. rubiginosum & Prob $>$ F \\
\hline Densidade básica $\left(\mathrm{g} / \mathrm{cm}^{3}\right)$ & 0,67 & 0,57 & - \\
\hline Freqüência de vasos (vasos $/ \mathrm{mm}^{2}$ ) & $(1-) 3(-8)$ & $(1-) 4(-12)$ & 0,0071 \\
\hline Diâmetro de vasos $(\mu \mathrm{m})$ & $(107-) 243(-368)$ & $(95-) 194(-286)$ & 0,0001 \\
\hline Comprimento de vasos $(\mu \mathrm{m})$ & $(306-) 566(-829)$ & $(320-) 549(-910)$ & 0,6560 \\
\hline Diâmetro das pontoações intervasculares $(\mu \mathrm{m})$ & (9-) $11(-15)$ & $(10-) 12(-17)$ & 0,2100 \\
\hline Comprimento das fibras $(\mu \mathrm{m})$ & $(621-) 961(-1212)$ & $(661-) 980(-1301)$ & 0,4862 \\
\hline Diâmetro das fibras $(\mu \mathrm{m})$ & $(12-) 19(-29)$ & $(8-) 17(-23)$ & 0,0269 \\
\hline Espes. da parede das fibras $(\mu \mathrm{m})$ & $(4-) 6(-10)$ & $(2-) 5(-8)$ & 0,0058 \\
\hline Freqüência de raios (raios/mm) & $(7-) 10(-14)$ & $(6-) 10(-14)$ & 0,0233 \\
\hline Altura dos raios $(\mu \mathrm{m})$ & $(123-) 247(-377)$ & $(61-) 196(-371)$ & 0,9215 \\
\hline Largura dos raios $(\mu \mathrm{m})$ & $(12-) 23(-48)$ & $(11-) 16(-23)$ & 0,0000 \\
\hline
\end{tabular}


firme (AM, PA), taxirana (RO), veludo (BA) (Carvalho 1994; Paula \& Alves 1997).

Com base no levantamento realizado conclui-se que as madeiras das duas variedades estudadas são muito utilizadas em comunidades rurais do sul do Maranhão, sendo a variedade subvelutinum a preferida entre os agricultores. A cachamorra-preta é mais utilizada para estacas, mourões e cercados, e a cachamorra-branca para lenha, carvão, cabo de ferramentas e peças longas. Esta afirmação está de acordo com o citado por B.A.S. Pereira (dados não publicados), Dias et al. (1992), Carvalho (1994), Lorenzi (1998). Apesar dos entrevistados terem informado que a cachamorra-branca cresce mais em
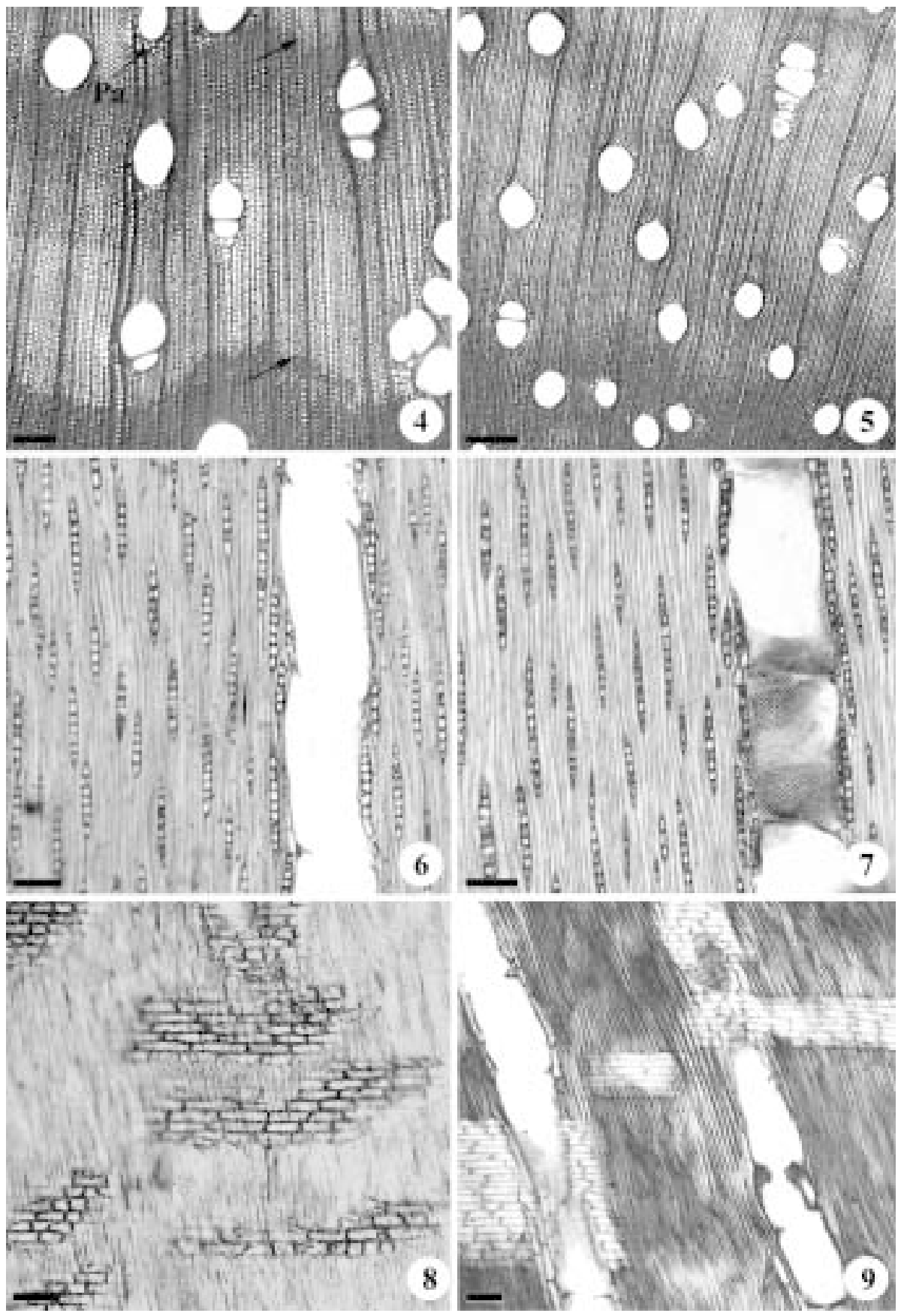

Figuras 4-9. Sclerolobium paniculatum Vog. 4, 6, 8. S. paniculatum var. subvelutinum Benth. 5, 7, 9. S. paniculatum var. rubiginosum (Mart. ex Tul.) Benth. 4-5. Seções transversais; (nota-se a porosidade difusa e vasos na maioria solitários). 4. As setas indicam as camadas de crescimento demarcadas pelo achatamento da parede das fibras. Barra $=100 \mu \mathrm{m}$. 5. Barra $=200 \mu \mathrm{m}$. 6-7. Seções longitudinais tangenciais; raios exclusivamente solitários. 8-9. Seções longitudinais radiais com raios homogêneos compostos por células procumbentes. $\mathrm{Pa}=$ parênquima axial. Barra $=100 \mu \mathrm{m}$. 


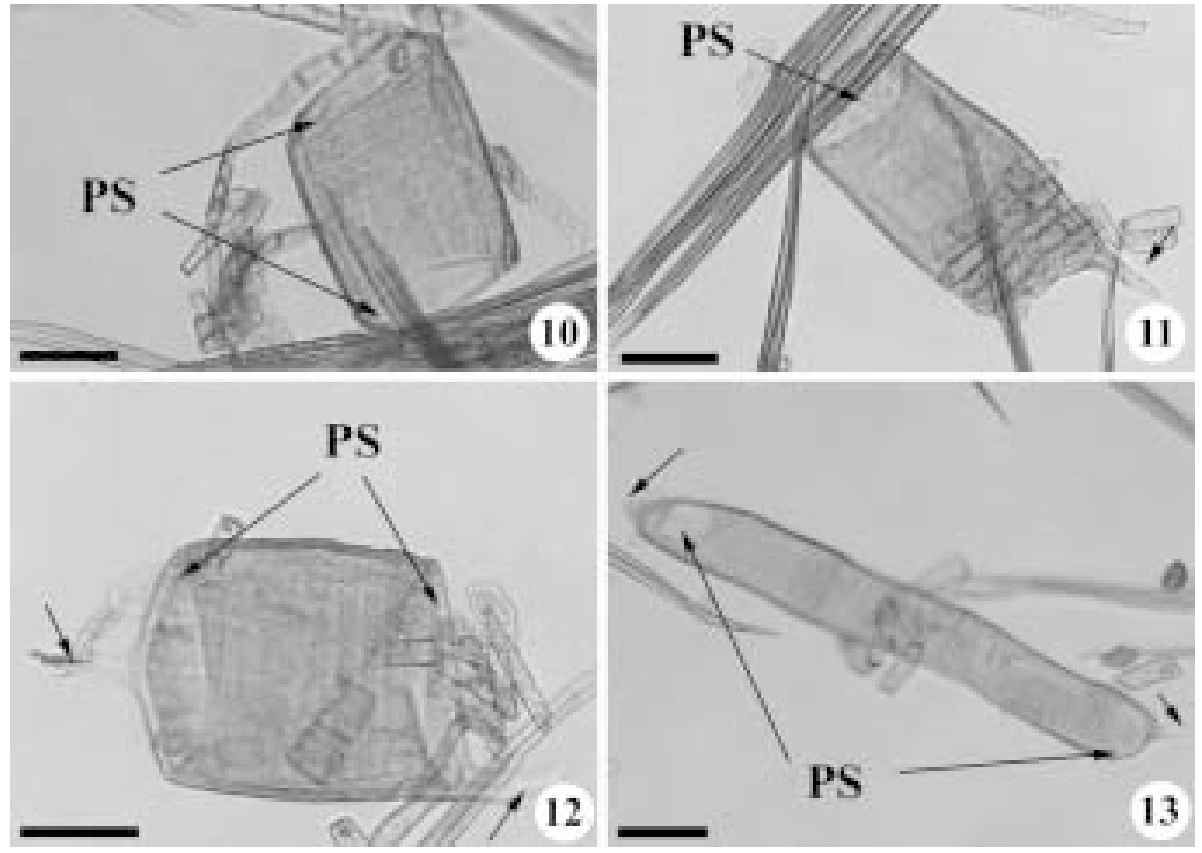

Figuras 10-13. Variação dos elementos de vasos de S. paniculatum Vog. em material dissociado. 10. Elemento de vaso sem apêndices em S. paniculatum var. subvelutinum Benth. 11. Elemento de vaso com apêndice em uma das extremidades (seta) em $S$. paniculatum var. rubiginosum (Mart. ex Tul.) Benth. 12-13. Elementos de vasos com apêndices nas duas extremidades (setas) em $S$. paniculatum var. rubiginosum . PS = placa de perfuração simples. Barra $=100 \mu \mathrm{m}$.

altura no campo quando comparada com a cachamorrapreta e que por esse motivo a madeira dessa variedade é utilizada para obtenção de peças longas, não houve diferença significativa $(\mathrm{p}<0,05)$ entre os valores de altura para as duas variedades. Também não houve diferença significativa $(\mathrm{p}<0,05)$ para o diâmetro das árvores entre as duas variedades.

Não foram encontradas informações na literatura quanto à durabilidade natural e a resistência mecânica da madeira das variedades estudadas, entretanto a maior densidade da madeira da cachamorra-preta, obtida no estudo, poderia estar relacionada com as melhores propriedades indicadas pelos agricultores da região.

As duas variedades apresentam camadas de crescimento irregularmente demarcadas por zonas fibrosas e porosidade difusa, informações já citadas por B.A.S. Pereira (dados não publicados) com as mesmas variedades ocorrentes no Distrito Federal, e por SUDAM (1981), Mainieri et al. (1983) e Mainieri \& Chimelo (1989) para o gênero Sclerolobium. A porosidade difusa da madeira é situação comum entre as dicotiledôneas brasileiras (Alves \& AngyalossyAlfonso 2000).

As diferenças anatômicas do lenho das variedades estão relacionadas apenas à variação da freqüência e diâmetro dos vasos, freqüência e largura dos raios e diâmetro e espessura da parede das fibras. A frequiência dos vasos foi comparativamente maior na variedade rubiginosum enquanto que os demais parâmetros foram maiores na variedade subvelutinum.

Neste estudo observou-se que houve diferença quanto à percentagem, freqüência, diâmetro e comprimento de vasos da madeira das variedades subvelutinum e rubiginosum quando comparadas com os resultados obtidos por B.A.S. Pereira (dados não publicados) para as mesmas variedades que ocorrem no Distrito Federal. As madeiras destas variedades, ocorrentes no Distrito Federal (B.A.S. Pereira, dados não publicados), apresentaram maior percentagem de vasos solitários e maior freqüência de vasos $(65 \mathrm{e}$ $62 \%$ e 5,79 e 6,49 vasos $/ \mathrm{mm}^{2}$ para as variedades subvelutinum e rubiginosum, respectivamente) e vasos de menor diâmetro e mais curtos (157 e 249 $\mu \mathrm{m}$ e 172 e $275 \mu \mathrm{m}$ para a madeira das variedades subvelutinum e rubiginosum, respectivamente), quando comparados com os valores obtidos neste estudo para as madeiras das variedades ocorrentes no sul do Maranhão. A falta de informações sobre os fatores edáficos e climáticos da região de estudo não permite discussão abrangente quanto às variações quantitativas observadas.

A presença de placas de perfuração simples e de 
pontoações guarnecidas são características da família Leguminosae, entretanto, Alves \& Angyalossy-Alfonso (2000) constataram fortes indicações da existência de relação entre pontoações guarnecidas e ambientes secos. As guarnições teriam papel importante na prevenção de embolias, aumentando a aderência das moléculas de água à parede das células (Carlquist 1982; 1983; 1988). A presença das pontoações guarnecidas nos vasos da madeira das variedades estudadas pode estar associada às condições secas do cerrado, uma vez que o cerrado é caracterizado por uma sazonalidade pluvial forte com invernos frios e secos, e apresenta solo compacto e seco nos primeiros metros de profundidade (Ferri 1979; Rizzini 1997; Franco 2002; Oliveira \& Marquis 2002).

Parênquima axial vasicêntrico e raios unisseriados
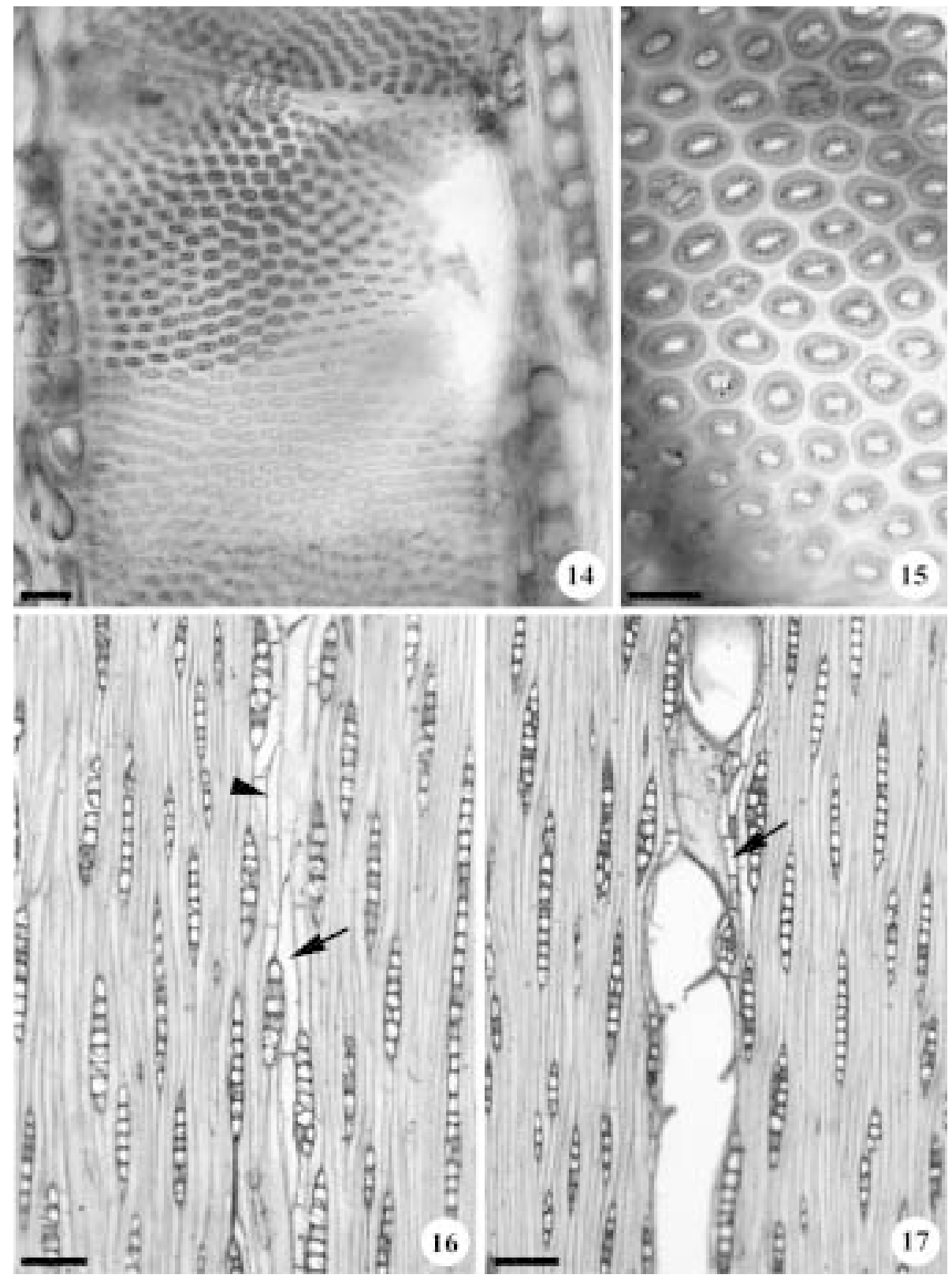

Figuras 14-17. Seções longitudinais tangenciais de S. paniculatum Vog. 14. S. paniculatum var. rubiginosum (Mart. ex Tul.) Benth. Pontoações intervasculares alternas, guarnecidas. 15. Detalhe da Figura 14 mostrando pontoações guarnecidas. 16-17. S. paniculatum var. rubiginosum. Seções longitudinais tangenciais mostrando séries parenquimáticas. 16. Séries parenquimáticas com duas células (seta) e três células (cabeça de seta). 17. Série parenquimática com quatro células (seta). Barras = $25 \mu \mathrm{m}(14) ; 10 \mu \mathrm{m}(15) ; 50 \mu \mathrm{m}(16,17)$. 
e homocelulares são característicos do gênero Sclerolobium (SUDAM 1981; Mainieri et al. 1983; Mainieri \& Chimelo 1989; B.A.S. Pereira, dados não publicados). Entretanto, os dados de percentagem de raios unisseriados obtidos por B.A.S. Pereira (dados não publicados) para as variedades subvelutinum e rubiginosum ocorrentes no Distrito Federal, 69\% e $80 \%$, respectivamente, são menores em relação aos aqui apresentados (99\%).

Apesar das fibras das duas variedades serem classificadas como finas a espessas segundo a classificação do IAWA Committee (1989), a madeira da variedade subvelutinum apresenta maior espessura da parede das fibras quando comparada com a madeira da variedade rubiginosum. A espessura da parede das fibras, a freqüência e diâmetro dos vasos, a quantidade de tecido parenquimático, além de outros fatores podem afetar a densidade da madeira e, conseqüentemente, sua durabilidade natural (Kollmann \& Coté 1968; Panshin \& De Zeeuw 1980; Zobel \& Buijtenen 1989).

Sugere-se que estudos onde sejam incluídas análises das características edáficas e variações climáticas do local de ocorrência das variedades sejam realizados, uma vez que as variedades estudadas podem ocorrer em ambientes diferentes das áreas de cerrado.

\section{Agradecimentos}

À UNESP, pela concessão da Bolsa do Programa de Apoio ao Estudante (PAE); à Dra. Vera T.R. Coradin e José A.A. Camargos do IBAMA, Brasília, pelo auxílio nas técnicas em anatomia da madeira; ao professor José Elias de Paula e colaboradores, do Departamento de Botânica da Universidade de Brasília, pela identificação das variedades; ao Genivaldo P. Gomes, pela ajuda na coleta do material de campo; a todos os agricultores que responderam ao questionário proposto.

\section{Referências bibliográficas}

Almeida, S.P. de; Proença, C.E.B.; Sano, S.M. \& Ribeiro, J.F. 1998. Cerrado: espécies vegetais úteis. Planaltina, EMBRAPA-CPAC.

Alves, E.S. \& Angyalossy-Alfonso, V. 2000. Ecological trends in the wood anatomy of some brazilian species. IAWA Journal 21(1):3-30.

Associação Brasileira de Normas Técnicas. 1940. Ensaios físicos e mecânicos da madeira. MB-26/40. Rio de Janeiro, ABNT.

Carlquist, S. 1982. Wood anatomy of Illcium (Illiciaceae): phylogenetical, ecological and functional interpretations. American Journal of Botany 69(10):1587-1598.
Carlquist, S. 1983. Wood anatomy of Onagraceae: further species; root anatomy; significance of vestured pits and allied structures in dicotyledons. Annals of the Missouri Botanical Garden 69(4): 755-769.

Carlquist, S. 1988. Comparative wood anatomy: systematic ecological and evolutionary aspects of dicotyledonean wood. Berlin, Springer-Verlag.

Carvalho, P.E.R. 1994. Espécies florestais brasileiras: recomendações silviculturais, potencialidades e usos da madeira. Colombo, EMBRAPA-CNPF.

Dias, L.E.; Jucksch, J.; Alvarez, V.H.; Barros, N.F. \& Brienza Junior, S. 1992. Formação de mudas de táxi-branco (Sclerolobium paniculatum Vogel): resposta a nitrogênio, potássio e enxofre. Revista Árvore 16(2): 135-143.

Diniz, J.A.F. 1982. A área centro-ocidental do nordeste. Recife, Sudene.

Ferri, M.G. 1979. Transpiração nos principais ecossistemas brasileiros e em espécies cultivadas no Brasil, Pp. 25-73. In: M.G. Ferri (ed.). Fisiologia Vegetal. São Paulo, EDUSP.

Franco, A.C. 2002. Ecophysiology of woody plants, Pp. 178-197. In: P.S. Oliveira \& R.J. Marquis (eds.). The cerrados of Brazil. New York, Columbia University Press.

Franke, I.L. 1999. Principais usos e serviços de árvores e arbustos promissores que ocorrem em pastagens no estado do Acre. Rio Branco, EMBRAPA.

Franklin, G.L. 1945. Preparation of thin sections of synthetic resins and wood-resin composites, and a new macerating method for wood. Nature 155(3924): 51.

Gerlach, D. 1984. Botanische mikrotechnik. Stuttgart, Georg Thieme Verlag.

International Association of Wood Anatomists Committee. 1989. List of microscopic features for hardwood identification. IAWA Bulletin 10(3): 220-332.

Kollmann, F. \& Côté Jr., W.A. 1968. Principles of wood science and technology. New York.

Kropp, U. 1972. Leitbündel. Mikrokosmos 61: 342-345.

Lorenzi, H. 1998. Árvores brasileiras: manual de identificação e cultivo de plantas arbóreas nativas do Brasil. $2^{a}$ ed., v.2. Nova Odessa, Editora Plantarum.

Mainieri, C.; Chimelo, J.P. \& Angyalossy-Alfonso, V. 1983. Manual de identificação das principais madeiras comerciais brasileiras. São Paulo, Instituto de Pesquisas Tecnológicas do Estado de São Paulo, PROMOCET.

Mainieri, C. \& Chimelo, J.P. 1989. Fichas de características das madeiras brasileiras. São Paulo, Instituto de Pesquisas Tecnológicas do Estado de São Paulo, 2a ed., Publicação IPT 1791.

Oliveira, P.S. \& R.J. Marquis. 2002. The cerrados of Brazil. New York, Columbia University Press.

Panshin, A.J. \& De Zeeuw, C. 1980. Textbook of wood technology. 4. ed. New York, McGraw-Hill Book Company.

Paula, J.E. \& Alves, J.L. 1997. Madeiras nativas. Brasília, Gráfica Gutemberg.

Ribeiro, J.F. \& Walter, B.M.T. 1998. Fitofisionomias do bioma cerrado. In: S.M. Sano \& S.P. Almeida (eds.). Cerrado: ambiente e flora. Planaltina, EMBRAPA-CPAC. 
Rizzini, C.T. 1997. Tratado de fitogeografia do Brasil: aspectos ecológicos, sociológicos e florísticos. Rio de Janeiro, Âmbito Cultural Edições Ltda.

Roeser, K.R. 1972. Die Nadel der Schwarzkiefer. Massenprodukt und Kunstwerk der Natur. Mikrokosmos 61: $33-36$.
Superintendência do Desenvolvimento da Amazônia. 1981. Madeiras da reserva florestal de Curuá-una estado do Pará: caracterização anatômica, propriedades gerais e aplicações. Belém, Ministério do Interior.

Zobel, B.J.; Buijtenen, J.P. van. 1989. Wood variation: its causes and control. Berlin, Springer-Verlag. 